\title{
Helmholtz Hamiltonian Mechanics Electromagnetic Physics Gaging generalizing mass-charge and charge-fields gage metrics to quantum relativity gage metrics
}

\begin{abstract}
Rajan Iyer
Environmental Materials Sciences Quantum Astrophysicist, Engineeringinc International Earth Global, Department of Physical Technological Sciences, Tempe, Arizona, United States of America. Email address: engginc@msn.com
\end{abstract}

\begin{abstract}
This article will continue ansatz gage matrix of Iyer Markoulakis Helmholtz Hamiltonian mechanics points' fields gage to Pauli Dirac monopole particle fields ansatz gage general formalism at Planck level, by constructing a Pauli Dirac Planck circuit matrix field gradient of particle monopole flow loop. This circuit assembly gage (PDPcag) that maybe operating at the quantum level, demonstrates the power of point fields matrix theoretical quantum general formalism of Iyer Markoulakis Helmholtz Hamiltonian mechanics transformed to Coulomb gage metrics, to form eigenvector fields of magnetic monopoles as well as electron positron particle gage metrics fields. Eigenvector calculations performed based on Iyer Markoulakis quantum general formalism are substituted for gage values of typical eigenvectors of dipolar magnetically biased monopoles with their conjugate eigenvectors, as well as eigenvector fields that are of the electron and positron particles. Then they are compiled to form combinatorial eigenvector matrix bundle of the monopole particle circuit field constructs assembly. Evaluation of this monopole particle fields matrix provided eigenvector fields results like $\operatorname{SUSY}\left(\begin{array}{cc}\mathbf{1} & \mathcal{\varepsilon} \\ \mathcal{E} * & \mathbf{1}\end{array}\right)$, having Hermitian quantum matrix with electron-positron annihilation alongside north and south monopoles collapsing to dipolar "stable" magnetism, representing electromagnetic gaging typical metrics fields. Applying experimental observations on magnetic poles with measuring magnetic forces John Hodge's results were showing asymmetrical pole forces; author has mathematically constructed asymmetriclstringslgagelmetrics to characterize electromagnetic gravity, putting together while integrating with stringmetrics gravity that author has been reporting in earlier published articles.

Physical Analysis with generalization of mass-charge and charge-fields gage metrics to quantum relativity gage metrics fields are proposed based on author's proof formalism paper providing derivational algorithmic steps, to determine gage parametric values within the equation of Coulomb gage. Vortex fields' wavefunctions and the scalar potential characterized by a function and a coupling constant having quantum density matrix together define the gage metrics quantifiable observable measurement physics of electron-positron cross-diagonal fields; contrastingly, diagonal terms of PDPcag matrix characterizes electron-positron particle eigenvector fields, while Hilbert Higgs mass metrics characterizes eigen-matter.
\end{abstract}


Author is already working with Christopher O’Neill about magic square symmetry configurations to quantitatively understand symmetry, structure, and the real space geometry that are expected to form out of vacuum quanta point fields' quantitative quantum general formalism theory of Iyer Markoulakis. In addition, author is currently collaborating with Manuel Malaver's astrophysical Einstein Minkowski modified space time metrics evaluations of the sense-timespace relativistic general metrics to have means to account for curving or shaping of spacetime topology of a five-dimensional sense-time-space. Manuel Malaver's specialization with modified Einstein Maxwell equations for modeling galaxies and stars cosmological physics, utilizing Einstein-Maxwell-Tolman- Schwarzschild and Reissner-Nordström spacetime and black holes theoretical formalisms have author of this paper collaboratively model quantum astrophysics of dark energy Star's theory with Einstein-Gauss-Bonnet gravity equations.

\section{Key Words: Physics, Helmholtz Hamiltonian Mechanics, stringmetrics,} Electromagnetism, Pauli Dirac Planck circuit like monopole particle assembly, Gage, asymmetriclstrings|gagelmetrics, Transforms, SUSY, Quantum Astrophysics.

\section{Introduction}

Point fields have been modeled applying Helmholtz decomposition matrix of gradient fields and rotational vortex fields, based on real time observational measurements with Ferrolens of a synthetic magnetic monopole assembly magneton [1,2]. Iyer Markoulakis Helmholtz Hamiltonian quantum mechanical general formalism derived [1] have been applied to solve practical problem of attraction and repulsion of entity point objects, specifically encountered in all electronic and magnetic entity forms, that will include monopoles within a dipole quagmire, by deriving algebraic equations [3], from the partial differential equations of general formalism [1\}. Graphic plots having input vortex signals and output sinusoidal signals showed bunching effects, suggestive of conversion of energy to condensed form like time-crystal physics, with quasi, fermionic, or bosonic type of particles, alongside possibility of existence of a superfluid condensate acting in essence as vacuum quanta quagmire [3]. Estimation of sizes of these entities to be typically $10^{-20} \mathrm{~m}$, that is many orders of magnitude less than the known sizes of fermions and Bohr atom, $10^{-18} \mathrm{~m}$ to $10^{-15} \mathrm{~m}$. Analytical interpretation of above computations showed that depending on monopole mass, entity sizes with zeropoint gradient energy of 1026 metric units, sizes of these entities may vary from values of $10^{-20} \mathrm{~m}$ or even lesser to $10^{-18} \mathrm{~m}$, especially due to microblackhole mechanism compressing monopole mass from $10^{-47} \mathrm{~kg}$ to $10^{-11} \mathrm{~kg}$. With entities propagating away from microblackhole, monopole mass value reducing closer to $10^{-47} \mathrm{~kg}$ with evolution of time, continuous propagation of generators assisted by quantum field photon mediators will be expected to create quarks, antiquarks, as well as gluons out of vacuum quanta [3].

Physical mathematical gaging mechanics to electromagnetic theory has gotten facilitated by ansatz formalism able to pull out observables with parameters of quantum density matrix 
operator eigenfunctions having general energy tensor fields, $\mathbf{E}$, functional commutator, $\mathbf{F}^{\mathbf{E}} \mathbf{t}$. with density matrix, $\boldsymbol{\rho}(\mathbf{t})$, influencing time event process. Time fields that are typical of microblackholes, shown to be analytically evidenceable from differential equations will determine these processes [4]. Iyer Markoulakis Helmholtz Hamiltonian general formalisms have been aptly converted to gage matrix [5], following physics literature procedures. Transformation of Helmholtz metrics to Coulomb gage, linking also Coulomb branch gage group with Hilbert series has also been quantifiably achieved having gradient fields converted to Coulomb gage, with rotational vortex fields branching to Hilbert gauge mass metrics of Higgs-Boson matter, and conforming to partial differential equations of vortex and the gradient fields obtained per Iyer Markoulakis original general formalism $[1,5]$. Vacuum gravitational solutions of the fields provided means to arrive at unitary determinant that will analytically project to having almost infinite extension of gauge matrix metrics, like stringmetrics construct showing typically charge asymmetry gage metrics [5]. Results of general formalism have been also discussed extensively with Physical analysis of particle physics gage matrix pointing to Dirac seas of electrons, monopoles with positrons, electron-positron annihilation leading to energy production, and the relativistic energy generating matter; quantum astrophysics gage metrix proposed mechanism of creation of neutrino antineutrino pair orthogonal to electron positron "curdling" planes, that may lead to the formation of protonic hydrogen of stars or orthogonally to these "curdling" planes muon particles, that are consistent with physics literature, have been elaborated further with concept of the vacuum monopoles posed to occur at extent of infinite cosmos $[1,3,5]$.

Gage conversions are quite useful to invariantly transfer information of fields of one type, like mechanics onto the fields of another type like electromagnetism; for example, Helmholtz Hamiltonian mechanics metrics quantifying mechanical fields can be gaged to Coulombic Hilbert metrics, representing Gilbertian and Amperian natures of electromagnetic fields [6, 7, 8]. Gauge transformations typically between possible gauges tend to form a Lie group, in general referred as symmetry group or the gauge group of the theory; Lie algebra of group generators quantifies a lie group [9]. Typical gauge transformation in general can be any formal, systematic transformation of the potentials that leaves the fields invariant, although in quantum theory it can be perhaps a bit more subtle than that because of the additional degree of freedom represented by the quantum phase $[9,10]$.

It is the application of Iyer Markoulakis general formalism [1] capable of gaging Helmholtz decomposition fields onto Pauli Dirac monopole particle fields [11] that is of concern here to model ansatz quantitatively general formalism at Planck level physics. Section 2 shows construct of a Pauli Dirac Planck circuit matrix field gradient of particle monopole flow loop. Section 2.1 configures application modeling using rules of Iyer Markoulakis Helmholtz Hamiltonian mechanics to evaluate eigenvector field bundle matrix of Pauli Dirac dipole biased monopoles+ electron-positron particle gaged field circuit, evaluating string gage metrics. Section 3 proceeds Physical Analysis with Results and Discussions, having Section 3.1 discussing by a brief note knowhow of generalizing mass-charge quantum metrics from general formalism of Iyer Markoulakis [1] and that of charge-fields obtained per Section 2 using proof formalism [4] and physics formalism [5] to define observable measurable gage metrics. Section 3.2 encompasses general relativistic time space sense symmetry shaping aspects with pressure matter gage 
metrics, as well as electro-magnetic gage metrics fields via quantum Helmholtz Hamiltonian mechanics formalized originally with theory of Iyer Markoulakis generalization provided [1], evaluating Coulomb gage out of fundamental proof formalism [4] in terms of pure state's coupling constant quantum density matrix with a function operator quantifying action vortex wavefunctions and the scalar potential of gradient up energy. In Section 3, these are eventually connected to a fundamental symmetry operational natural mechanism of prime numbers' factorization evolving magic square symmetry capable of defining structure of real space geometry in Section 3.3. Astrophysical Einstein Minkowski modified space time metrics evaluations are outlined. Section 4 summarizes quantum circuit assembly, eigenvector of the dipolar biased monopoles, and electron-positron particles with symmetry operations giving SUSY like field matrix and theoretical evaluation technique for algebra of Coulomb gage, the magic square symmetry, and astrophysical sense-time-space relativistic general metrics to obtain quantum relativistic grand unification physics.

\section{Theoretical Results Physics Gaging Formalism}

\section{Schematic of matrix Pauli Dirac Planck circuit}

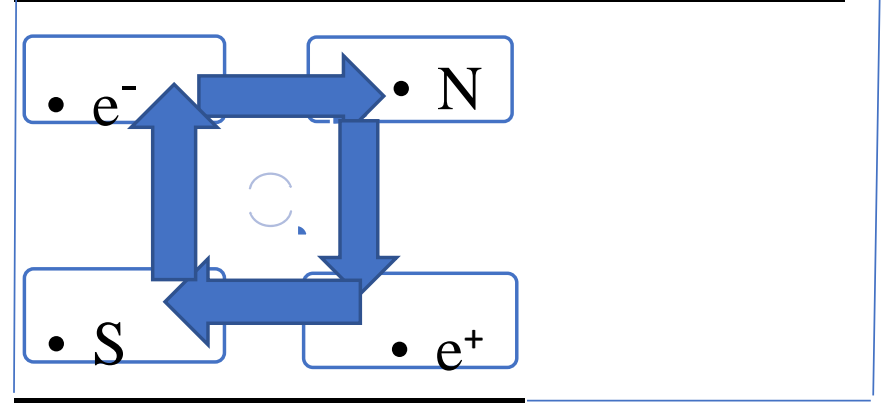

$\mathrm{e}^{-}$: electron, and $\mathrm{e}^{+}$: positron particles; $\mathrm{N}$ : north, and $\mathrm{S}$ : south monopoles - flow of arrow shows gradient vortex matrix circuit.

\subsection{Configuring application matrix Iyer Markoulakis Helmholtz Hamiltonian mechanics monopole particle gaged field circuit ext}

Substituting field equivalents of gradient monopole rotational particle Iyer Markoulakis Helmholtz Hamiltonian mechanics theory [1] of algebra for Pauli Dirac Planck circuit matrix, get

$\left(\begin{array}{ll}\widehat{\varepsilon}_{\mathrm{r}, \mu \nu} & \hat{\boldsymbol{\varepsilon}}_{\mathrm{g}}^{\mu \nu} \\ \hat{\varepsilon}_{\mathrm{g}, \mu \nu} & \widehat{\varepsilon}_{\mathrm{r}}^{\mu \nu}\end{array}\right)=>::<=\left(\begin{array}{cc}\widehat{\varepsilon}_{\mathrm{e}-} & \widehat{\varepsilon}_{\mathrm{n}} \\ \widehat{\varepsilon}_{\mathrm{s}} & \widehat{\varepsilon}_{\mathrm{e}}^{+}\end{array}\right)$

with $\widehat{\boldsymbol{\varepsilon}}_{\mathbf{g}, \boldsymbol{\mu \nu}}$ : gradient converging field gaged to $\widehat{\boldsymbol{\varepsilon}}_{\mathbf{s}}$ : south monopole field; $\widehat{\boldsymbol{\varepsilon}}_{\mathbf{g}, \boldsymbol{\mu \nu}}$ : gradient diverging field gaged to $\widehat{\boldsymbol{\varepsilon}}_{\mathbf{n}}$ : north monopole field; similarly, $\widehat{\boldsymbol{\varepsilon}}_{\mathbf{r}, \boldsymbol{\mu \nu}}$ : rotational vortex converging field gaged 
to $\widehat{\boldsymbol{\varepsilon}}_{\mathbf{e}-\mathbf{s}}$ : electron particle metrics field, and then $\hat{\boldsymbol{\varepsilon}}_{\mathbf{r}}^{\mu \nu}$ : rotational vortex diverging field gaged to $\widehat{\boldsymbol{\varepsilon}}_{\mathbf{e}}^{+}$: positron particle metrics field; together they are forming field matrix.

Eigenvector calculations performed already in Iyer Markoulakis general formalism [1] are substituted for gage values. $\left|\boldsymbol{\lambda}_{\mathbf{d n}}\right\rangle$ : eigenvector of dipolar biased north monopole (having proximity of the north and south monopoles making them act like dipole having bias to the closer monopole); similarly, $\left|\boldsymbol{\lambda}_{\mathbf{d s}}\right\rangle$ : eigenvector of dipolar biased south monopole; $\mid \boldsymbol{\lambda}_{\mathbf{d n}} * \boldsymbol{\gamma}$ and $\left|\boldsymbol{\lambda}_{\mathbf{d s}} *\right\rangle$ are conjugate eigenvectors of $\left|\boldsymbol{\lambda}_{\mathbf{d n}}\right\rangle$ and $\left|\boldsymbol{\lambda}_{\mathbf{d s}}\right\rangle$ respectively, while $\left|\boldsymbol{\lambda}_{\mathrm{e}-}\right\rangle$ and $\left|\boldsymbol{\lambda}_{\mathrm{e}+}\right\rangle$ are eigenvectors of the electron and positron particles, following rules of Iyer Markoulakis general formalism linking them to the appropriate field matrix vectors' quanta [1].

$$
\begin{aligned}
& \left|\lambda_{\mathrm{dn}}\right\rangle=\left(\begin{array}{c}
-\left(\hat{\varepsilon}_{\mathrm{s}} \hat{\varepsilon}_{\mathrm{n}}\right)^{\wedge} 0.5 \\
\hat{\varepsilon}_{\mathrm{n}}
\end{array}\right) ;\left|\lambda_{\mathrm{ds}}\right\rangle=\left(\begin{array}{c}
\hat{\varepsilon}_{\mathrm{s}} \\
-\left(\hat{\varepsilon}_{\mathrm{s}} \hat{\varepsilon}_{\mathrm{n}}\right)^{\wedge} 0.5
\end{array}\right) ;\left|\lambda_{\mathrm{ds}}\right\rangle=\left(\begin{array}{c}
\hat{\varepsilon}_{\mathrm{s}} \\
\left(\hat{\boldsymbol{\varepsilon}}_{\mathrm{s}} \hat{\varepsilon}_{\mathrm{n}}\right)^{\wedge} 0.5
\end{array}\right) ;\left|\lambda_{\mathrm{dn}}\right\rangle=\left(\begin{array}{c}
\left(\hat{\varepsilon}_{\mathrm{s}} \hat{\varepsilon}_{\mathrm{n}}\right)^{\wedge} 0.5 \\
\hat{\varepsilon}_{\mathrm{n}}
\end{array}\right) \\
& \left|\lambda_{\mathrm{e}-}\right\rangle=\left(\begin{array}{l}
1 \\
0
\end{array}\right) ;\left|\lambda_{\mathrm{e}+}\right\rangle=\left(\begin{array}{l}
0 \\
1
\end{array}\right)
\end{aligned}
$$

Equation (1) gives relevant field 2x2 gage matrix, while Equation (2) gives eigenvector fields of these monopole particle circuit components specifically characterizing each entity of monopole and particle in detail completely. With this algebra, we can compile matrix assembly with combinatorial eigenvector bundle of the monopole particle circuit. We denote

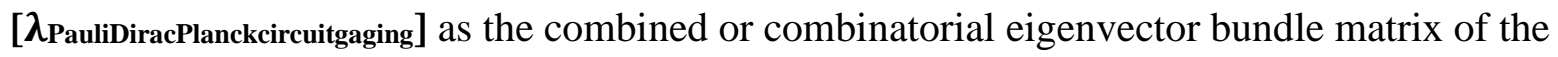
$\mid \boldsymbol{\lambda} \mathbf{e}->$ : electron particle eigenvector, $\mid \boldsymbol{\lambda} \mathbf{e}+>$ : positron particle eigenvector, $\mid \boldsymbol{\lambda} \mathbf{d n} * \mathbf{n}$ : dipolar biased north monopole conjugate eigenvector, and then that $|\lambda \mathbf{d} \mathbf{s} *\rangle$ : dipolar biased south monopole conjugate eigenvector, altogether forming $2 \times 2$ eigenvector assembly matrix of eigenvector Planck circuit gaging Pauli Dirac particle monopole quantum fields. These are the constructs shown below.

$[\lambda$ PauliDiracPlanckcircuitgaging $]=\left(\begin{array}{ll}\mid \lambda \mathbf{e}-> & \mid \lambda \mathbf{d n} *> \\ \mid \lambda \mathbf{d s} *> & \mid \lambda \mathbf{e}+>\end{array}\right)$

Substituting these eigenvectors' values from Equation (2) to Equation (3), we arrive thus:

$$
\left[\lambda_{\text {PauliDiracPlanckcircuitgaging }]}=\left(\begin{array}{cc}
\left(\begin{array}{l}
1 \\
0
\end{array}\right) & \left(\begin{array}{c}
\left(\hat{\varepsilon}_{\mathrm{s}} \hat{\boldsymbol{\varepsilon}}_{\mathrm{n}}\right)^{\wedge} 0.5 \\
\hat{\boldsymbol{\varepsilon}}_{\mathbf{n}}
\end{array}\right) \\
\left(\begin{array}{c}
\hat{\boldsymbol{\varepsilon}}_{\mathrm{s}} \\
\left(\hat{\boldsymbol{\varepsilon}}_{\mathrm{s}} \widehat{\boldsymbol{\varepsilon}}_{\mathrm{n}}\right)^{\wedge} 0.5
\end{array}\right) & \left(\begin{array}{l}
0 \\
1
\end{array}\right)
\end{array}\right)\right.
$$

Symmetry operations will lead to collapse of above matrix, because of the zero of $\left(\begin{array}{l}1 \\ 0\end{array}\right)$ canceling $\hat{\boldsymbol{\varepsilon}}_{\mathbf{s}}$ of $\left(\begin{array}{c}\hat{\boldsymbol{\varepsilon}}_{\mathbf{s}} \\ \left(\hat{\boldsymbol{\varepsilon}}_{\mathbf{s}} \hat{\boldsymbol{\varepsilon}}_{\mathbf{n}}\right)^{\wedge} 0.5\end{array}\right)$ and similarly 0 of $\left(\begin{array}{l}0 \\ 1\end{array}\right)$ canceling $\hat{\boldsymbol{\varepsilon}}_{\mathbf{n}}$ of $\left(\begin{array}{c}\left(\hat{\boldsymbol{\varepsilon}}_{\mathbf{s}} \hat{\boldsymbol{\varepsilon}}_{\mathbf{n}}\right)^{\wedge} 0.5 \\ \hat{\boldsymbol{\varepsilon}}_{\mathbf{n}}\end{array}\right)$, effectively with overlapping elemental metrics field actions. Resultant will give eigenvector Pauli Dirac Planck circuit gaging assembly matrix simplified to that below: 
$\left[\boldsymbol{\lambda}_{\text {PauliDiracPlanckcircuitgaging }}\right]=\left(\begin{array}{cc}\mathbf{1} & \left(\hat{\boldsymbol{\varepsilon}}_{\mathbf{s}} \hat{\boldsymbol{\varepsilon}}_{\mathbf{n}}\right)^{\wedge} 0.5 \\ \left(\hat{\boldsymbol{\varepsilon}}_{\mathbf{s}} \hat{\boldsymbol{\varepsilon}}_{\mathbf{n}}\right)^{\wedge} 0.5 & \mathbf{1}\end{array}\right)$

We can evaluate matrix in Equation (5); cross-diagonals are conjugate elements, same magnitude

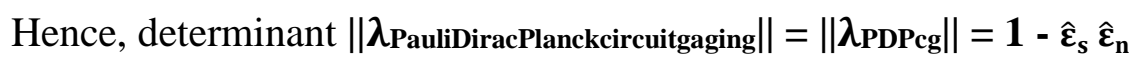

If $\widehat{\boldsymbol{\varepsilon}}_{\mathbf{n}}=\hat{\boldsymbol{\varepsilon}}$ and $\widehat{\boldsymbol{\varepsilon}}_{\mathbf{s}}=\widehat{\boldsymbol{\varepsilon}}^{*}$, then $\left\|\lambda_{\text {PDPcg }}\right\|=\mathbf{1}-\mathfrak{\varepsilon}^{\mathbf{2}}$, with $\hat{\boldsymbol{\varepsilon}}_{\mathbf{n}}$ and $\widehat{\boldsymbol{\varepsilon}}_{\mathbf{s}}=\mathcal{\varepsilon}$, having same scalar value; this equation otherwise may then be written like quadratic equation form:

$\mathcal{E}^{2}+\left\|\lambda_{\text {PDPcg }}\right\|-1=0$, giving that: $\mathcal{E}=+/-\left(1-\left\|\lambda_{\text {PDPcg }}\right\|\right)^{0.5} \quad \mathcal{E}^{*}$

We can also in general write having $\hat{\boldsymbol{\varepsilon}}_{\mathbf{n}}=\hat{\boldsymbol{\varepsilon}}$ and $\hat{\boldsymbol{\varepsilon}}_{\mathbf{s}}=\widehat{\boldsymbol{\varepsilon}}^{*}$, giving the eigenvector matrix:

\section{$\left[\lambda_{\text {PauliDiracPlanckcircuitgaging }}\right]=\left[\lambda_{\text {PDPcg }}\right]=\left(\begin{array}{cc}1 & \varepsilon \\ \varepsilon * & 1\end{array}\right)$}

Here, [ $\boldsymbol{\lambda}_{\text {PDPcg] }}$ matrix Equation (8) is like SUSY [12], having Hermitian quantum matrix [13, 14]. With electron-positron annihilation alongside monopoles north and south collapsing to dipolar "stable" magnetism, that is like stringmetrics gage [5]; however, in this case it will be like electromagnetic gaging fields [6-10].

Recent experiments with John Hodge [15] show that forces of south poles are slightly stronger than the north poles, that in our context will mean than $\hat{\varepsilon}_{\mathrm{s}}>\hat{\varepsilon}_{\mathrm{n}}$ slightly. Therefore, $\left[\lambda_{\text {PDPcg }}\right]$ will have asymmetry metrics, thereby asymmetriclstringslgagelmetrics, having nonHermitian quantum matrix [16-18]. This will point to anisotropic asymmetric eccentric precession with electromagnetic gaging fields [19]. Together with stringmetrics gravity [4] it will then constitute electromagnetic gravity [20].

\section{Physical Analysis with Results and Discussions}

\subsection{Brief note about generalizing mass-charge and charge-fields gage metrics to quantum relativity gage metrics}

Generalizing both gage metrics to get quantum relativity gage unitary metrics will have to undergo overall scheme:

masslchargelgagelquantumlmetrics

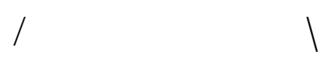

Iyer\Markoulakis\general\formalism\metrics => quantum\relativity\gagelunitary\metrics

quantumlchargelgagelmetricslfields 
This will then involve comparing Pauli Dirac Planck circuit gage $[\lambda$ PauliDiracPlanckcircuitgaging $]=\left(\begin{array}{cc}\mathbf{1} & \boldsymbol{E} \mathbf{d n} * \\ \mathcal{E d s} * & \mathbf{1}\end{array}\right)$ with [Coulomb Hilbert gage] $\left(\begin{array}{cc}\widehat{\mathbf{M}}_{\mathbf{r}, \boldsymbol{\mu \nu}} & \widehat{\mathbf{G}}_{\mathbf{g}}^{\mu \nu} \\ \widehat{\mathbf{G}}_{\mathbf{g}, \mu \nu} & \widehat{\mathbf{M}}_{\mathbf{r}}^{\mu \nu}\end{array}\right)(9)$ [5]. [Coulomb Hilbert gage] is essentially the masslchargelgagelquantumlmetrics, while

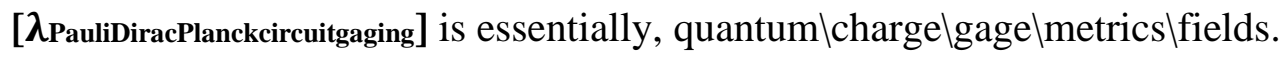

We will have to examine general formalism Coulomb Hilbert gage metrics shaping spacetime symmetry alongside Pauli Dirac Planck circuit electro-magnetic gage metrics fields, then put them together to get generalism unitary gage quantum\relativitylgagelunitary\metrics. Per physics [5] Coulomb gage, $\widehat{\mathbf{G}}$, may be algorithmically evaluated having proof mathematical matrix [4] per procedural scheme, showing scalar potential, $\mathbf{V}=\left\|\boldsymbol{\nabla} \quad \mathbf{E}_{\mathbf{g}}^{\mu v}\right\|$, related to gage metrics fields of Pauli Dirac Planck circuit gage. $\{\mathfrak{E d n} *, \mathcal{E d} *\}$ refer to quantum magnetic monopolar cross-diagonal fields, whereas $\left\{\widehat{\mathbf{G}}_{\mathrm{g}, \mu \nu}, \widehat{\mathbf{G}}_{\mathrm{g}}^{\mu \nu}\right\}$ refer to Coulomb quantum gage particle metrics per matrix formulation: $\widehat{\mathbf{G}}=\left(\left\langle\boldsymbol{\Psi}_{\mu}(\mathbf{t}) \mid \Psi^{\mu}(\mathbf{t})\right\rangle\right)^{-1}\left\|\boldsymbol{\nabla} \quad \mathbf{E}_{\mathrm{g}}^{\mu \nu}\right\| \boldsymbol{\rho}(\mathbf{t})$ of electron-positron crossdiagonal fields. Similarly, "1" s refers to particle diagonal eigenvector fields with Pauli Dirac Planck circuit gage, whereas $\left\{\widehat{\mathbf{M}}_{\mathbf{r}, \boldsymbol{\mu \nu}}, \widehat{\mathbf{M}}_{\mathbf{r}}^{\boldsymbol{\mu \nu}}\right\}$ refer to Hilbert Higgs metrics gage diagonal eigenmatter. Hence non-Hermitian CPT physics will have to consider these aspects while generalizing both gages - matter and fields, having diagonal terms constituting pressure matter gage metrics, cross-diagonal terms constituting electro-magnetic gage metrics fields.

\subsection{Pressure matter gage metrics shaping spacetime symmetry and electro-magnetic gage metrics fields energy quanta point}

To consider about pressure matter gage metrics, shaping sense time space symmetry aspects will have to be quantified applying relativistic Einstein metric of the Minkowski space time equation systems: $\mathbf{d s}^{2}=-\mathbf{d t}^{2}+\mathbf{d x}^{2}+\mathbf{d y}^{2}+\mathbf{d z}^{2}$ in Cartesian coordinates; in spherical

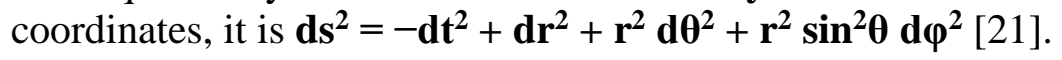

Rewriting in the vacuum continuum form in general metric form:

$Y=d s^{2}-g_{1 x} d x^{2}-g_{1 y} d y^{2}-g_{1 z} d z^{2}+g_{11 d t^{2}}$

Equation (10) having $\left\{\mathbf{g}_{\mathbf{1 x}}, \mathbf{g}_{\mathbf{1 y}}, \mathbf{g}_{\mathbf{1 z}}, \mathbf{g}_{\mathbf{1 t}}\right\}$ relativistic general metrics thus will account for curving or shaping of the $[\mathbf{x}, \mathbf{y}, \mathbf{z}, \mathbf{t}]$ spacetime topology, generalizing modification of functional parametric symmetric factor, $\mathrm{Y}=0$ which will correspond to vacuum solution with zero sense, if we have a five-dimensional sense time space universe [22]; while, vacuum monopoles probably occur infinitely with having universal vacuum quanta cosmos extent [23]. There are many astrophysical equations, modifying on Einstein-Maxwell spacetime fields equations to evaluate these metrics, like which author has coauthored collaborative paper of astrophysicist Manuel Malaver [24], that will be extensively considered later.

To consider about electro-magnetic gage metrics fields acting energy on quanta point fields, theoretical general formalism with Iyer Markoulakis Helmholtz Hamiltonian mechanics 
quantum field metrix will be applicable exactly [1]. Problem solving with gage equation: $\widehat{\mathbf{G}}=$ $\left(<\Psi_{\mu}(\mathbf{t})\left|\Psi^{\mu}(\mathbf{t})\right\rangle\right)^{-\mathbf{1}}\left\|\boldsymbol{\nabla} \quad \mathbf{E}_{\mathrm{g}}^{\mu \nu}\right\| \boldsymbol{\rho}(\mathbf{t})$, derived from mathematical physics first principle's proofing technique [4] have been successfully adapted to physics formalism with Iyer Markoulakis Helmholtz Hamiltonian mechanics transformed to Coulomb gage [5]. In this respect, Iyer Markoulakis theory [1] has specifically concentrated on quantifying Helmholtz decomposition fields analysis of vortex dynamics that have been observed by macro-scale synthetic magnetic monopole assembly experimental technique using Ferrocell [2]. This extensive quantum model ansatz formalism provided eigenvectors, wavefunctions, as well as partial differential equations characterizing zero-point gradient fields and point microblackhole vortex fields' mechanics that have been then converted to Coulomb gage to equivalently characterize electromagnetic point quanta [5]. In the above equation of Coulomb gage: $\widehat{\mathbf{G}}=\left(\left\langle\boldsymbol{\Psi}_{\boldsymbol{\mu}}(\mathbf{t}) \mid \boldsymbol{\Psi}^{\mu}(\mathbf{t})\right\rangle\right)^{-\mathbf{1}}\left\|\boldsymbol{\nabla} \quad \mathbf{E}_{\mathbf{g}}^{\boldsymbol{\mu \nu}}\right\| \boldsymbol{\rho}(\mathbf{t})$, with wavefunctions as a function of time, $\boldsymbol{\Psi}(\mathbf{t})$, with upper and the lower indices quantifying down and up vortex fields; gradient up energy, $\left\|\nabla \quad \mathbf{E}_{\mathbf{g}}^{\mu v}\right\|=\mathrm{V}$, the scalar potential; and $\boldsymbol{\rho}(\mathbf{t})=$ quantum density matrix, typically representing pure state's like coupling constant in general relativity [25]; overall we may also define a function operator, f quantifying $\left(\left\langle\Psi_{\mu}(\mathbf{t}) \mid \Psi^{\mu}(\mathbf{t})\right\rangle\right)^{-1}\left\|\boldsymbol{\nabla} \quad \mathbf{E}_{\mathrm{g}}^{\mu \boldsymbol{v}}\right\|$ considered as transforming micro to macro parametrically $\boldsymbol{\rho}(\mathbf{t})$ to $\widehat{\mathbf{G}}$. Together they all fully define the gage metrics quantifiable observable measurement physics $[4,5]$. To understand structural geometry formed from vacuum quanta out of point fields' quantitative theory $[1,5]$, it is presently required to grapple symmetry, structure, and formation of real space with knowledge of magic square symmetry [26] inherently naturally originating with mechanism characteristics of fundamental processes of prime numbers' factorization. We will examine this extensively in later sections as well as within subsequent papers.

\subsection{Magic square symmetry with processes of prime numbers' factorization natural operational mechanism}

Vacuum quanta may have only random scatter points patterns with fabric of the space [1, 5] in primordial universe. Research extensively performed about magic square symmetry and how that may represent natural mechanism with which point patterns align to form crystals have been explored quite recently, especially with Christopher O'Neill's simulations to study, for example, the typical patterns of the 48 particles of the DGO Standard Model assigned vertices in the 288-cell, as part of Quantum Physics project with Magic Squares, the Weak Force and the Higgs Mechanism [26]. Whereas $0->\widehat{\mathbf{M}}$, the diagonal Hilbert Higgs metrics [5], perhaps quantify Higgs mechanistic field operator generator, signifying action to matter inertia effectively operating with gravitational field moving from vacuum to matter, $\mathbf{M}$ in general will represent characterizing Helmholtz transformation symplectics to Higgs field, having subsequent Higgs mechanism to originate God particle giving flavor mass particle Higgs Boson system [27]. Additionally, this author's conjecture with recent quantum modeling articles $[1,3,4,5]$ lead to proposition that prime numbers' factorization of these random pop-up particles within vacuum $[1,28,29]$ will be a natural operational mechanism. Based on that are seen in the symmetrical 
universal map with Hubble Space Telescope, theoretically corresponding to the Emmy Noether's theorem of symmetry principle, discussed elsewhere [1], these can provide us an intuition that there may be inherent quantum asymmetry and there is driving force to attain symmetrical universe. However, only matter particle arrangements within the typical form of magic square symmetries are possible and that rotations/revolutions of matter are necessary to have group symmetry, also that in the process of which it will have to fractionate to fractal elements. Hence the scatter distributions of constellations, galaxies, stars, planets, and satellites make sense thereby, with also periodic lattice arrangement of atoms, micro-crystals, as well as macrocrystals, with even supersymmetry $[12,13]$. Quite detailed study about magic squares group symmetries revealing natural mechanisms operating will be revealed by articles continuing with this paper. We will strive to achieve understanding with knowledge of magic square symmetry mathematical physics revealing symmetry, structure, and formation of a natural operational real space geometry.

\subsection{Astrophysical Einstein Minkowski modified space time metrics evaluations}

Malaver et al have examined Einstein-Maxwell-Tolman- Schwarzschild and ReissnerNordström spacetime and black holes with theoretical complete formalisms [30]. Author of this paper has also coauthored paper on quantum astrophysics with Malaver et al [24] to model dark energy Star's theory based on Einstein-Gauss-Bonnet gravity equations. Highly detailed study about magic squares group symmetries revealing natural mechanisms operating will be revealed by articles continuing with this paper. Our goal will be to achieve derivation of the sense-timespace metrics within Equation (10) having $\left\{\mathbf{g}_{1 \mathbf{x}}, \mathbf{g}_{\mathbf{1 y}}, \mathbf{g}_{\mathbf{1 z}}, \mathbf{g}_{\mathbf{1 t}}\right\}$ relativistic general metrics. Thus, we will obtain quantitative means to account for curving or shaping of the $[\mathbf{x}, \mathbf{y}, \mathbf{z}, \mathbf{t}]$ spacetime topology, generalizing modifying parametric symmetric factor, with the function $\mathrm{Y}$ corresponding to sense solution of five-dimensional sense time space universe [22, 31]. These astrophysical equations, modifying on Einstein-Maxwell spacetime fields equations evaluating these metrics will be extensively considered as continuing articles of the present paper.

\section{Summary conclusions}

Author has presented configuration of Pauli Dirac Planck circuit assembly gage (PDPcag) that maybe operating at the quantum level, typically consistent with application of point fields matrix theoretical quantum general formalism of Iyer Markoulakis Helmholtz Hamiltonian mechanics transformed metrics with Coulomb gage, to form eigenvector fields of magnetic monopoles as well as electron positron particle fields metrics $\left(\begin{array}{ll}\widehat{\boldsymbol{\varepsilon}}_{\mathrm{r}, \mu \nu} & \widehat{\boldsymbol{\varepsilon}}_{\mathbf{g}}^{\mu v} \\ \widehat{\boldsymbol{\varepsilon}}_{\mathrm{g}, \boldsymbol{\mu \nu}} & \widehat{\boldsymbol{\varepsilon}}_{\mathbf{r}}^{\mu \nu}\end{array}\right)=>::<=\left(\begin{array}{cc}\widehat{\boldsymbol{\varepsilon}}_{\mathbf{e}-} & \widehat{\boldsymbol{\varepsilon}}_{\mathbf{n}} \\ \widehat{\boldsymbol{\varepsilon}}_{\mathbf{s}} & \hat{\boldsymbol{\varepsilon}}_{\mathbf{e}}^{+}\end{array}\right)$.

Eigenvector calculations performed based on Iyer Markoulakis general formalism [1] are substituted for gage values of those eigenvectors of dipolar biased monopoles as well as their 
conjugate eigenvectors. Similar procedures then yielded eigenvectors of the electron and positron particles, following rules of Iyer Markoulakis general formalism linking them to the appropriate field matrix vectors quanta. Relevant field $2 \times 2$ gage matrix of monopole particle circuit components' characteristics, specifically with each entity in detail completely were compiled to form combinatorial eigenvector bundle of the monopole particle circuit matrix constructs assembly [ $\lambda$ PauliDiracPlanckcircuitgaging] $=\left(\begin{array}{cc}\left(\begin{array}{l}1 \\ 0\end{array}\right) & \left(\begin{array}{c}\left(\hat{\boldsymbol{\varepsilon}}_{\mathbf{s}} \hat{\boldsymbol{\varepsilon}}_{\mathbf{n}}\right)^{\wedge} 0.5 \\ \hat{\boldsymbol{\varepsilon}}_{\mathbf{n}}\end{array}\right) \\ \left(\begin{array}{c}\widehat{\boldsymbol{\varepsilon}}_{\mathbf{s}} \\ \left(\hat{\boldsymbol{\varepsilon}}_{\mathbf{s}} \widehat{\boldsymbol{\varepsilon}}_{\mathbf{n}}\right)^{\wedge} 0.5\end{array}\right) & \left(\begin{array}{l}0 \\ 1\end{array}\right)\end{array}\right)$ simplifying with symmetry operations to matrix collapsing resultant eigenvector PDPcag of $\left(\begin{array}{cc}\mathbf{1} & \left(\hat{\boldsymbol{\varepsilon}}_{\mathbf{s}} \hat{\boldsymbol{\varepsilon}}_{\mathbf{n}}\right)^{\wedge} 0.5 \\ \left(\hat{\boldsymbol{\varepsilon}}_{\mathbf{s}} \widehat{\boldsymbol{\varepsilon}}_{\mathbf{n}}\right) *^{\wedge} 0.5 & \mathbf{1}\end{array}\right)$. Evaluation of this monopole-particle fields' matrix provided eigenvector matrix results like $\operatorname{SUSY}\left(\begin{array}{cc}\mathbf{1} & \boldsymbol{\varepsilon} \\ \varepsilon * & \mathbf{1}\end{array}\right)$, having Hermitian quantum matrix with electron-positron annihilation alongside monopoles north and south collapsing to dipolar "stable" magnetism, that is like stringmetrics, that in this case, representing electromagnetic gaging fields. Modifying this matrix with experimental observations with magnetic field measurements of macro-magnet-poles, that John Hodge originally has conducted, showing the forces of south poles having slightly stronger force than the north poles, eigenvector bundle will have asymmetriclstrings gagelmetrics, having nonHermitian quantum matrix that will point to anisotropic asymmetric eccentric precession with electromagnetic gaging fields. We saw a way to unify electromagnetism with gravity with these gravitational stringmetrics and electromagnetic eigenvector matrix.

Physical Analysis with generalization of mass-charge and charge-fields gage metrics to quantum relativity gage metrics fields has been also proposed, based on author's proof formalism paper providing precise derivational algorithmic steps, to determine gage parametric values within the equation of Coulomb gage: $\widehat{G} \quad=\left(\left\langle\Psi_{\mu}(\mathbf{t}) \mid \Psi^{\mu}(\mathbf{t})\right\rangle\right)^{-1}\left\|\nabla \quad E_{g}^{\mu v}\right\| \rho(t)=\mathbf{f} . \rho(t)$. These fundamental mathematical physics proof derivation of the wavefunctions of the vortex fields and the scalar potential characterized by the function, $\mathrm{f}$, and pure state's like coupling constant having quantum density matrix, $\boldsymbol{\rho}(\mathbf{t})$ together fully define the gage metrics quantifiable observable measurement physics of electron-positron cross-diagonal fields - "1"s of the PDPcag matrix refer to electron-positron particle diagonal eigenvector fields with Pauli Dirac Planck circuit gage, whereas $\left\{\widehat{\mathbf{M}}_{\mathbf{r}, \boldsymbol{\mu \nu}}, \widehat{\mathbf{M}}_{\mathbf{r}}^{\mu \boldsymbol{v}}\right\}$ of Hilbert Higgs metrics matrix gage diagonal refer to eigenmatter, requiring non-Hermitian CPT physics will have to be considered to generalize diagonal terms constituting pressure matter gage metrics and cross-diagonal terms constituting electromagnetic gage metrics fields.

Author is already working to have evaluation of pressure matter gage metrics shaping spacetime symmetry and electro-magnetic gage metrics fields energy quanta point. In this respect, magic square symmetry with processes of prime numbers' factorization natural operational mechanism alongside astrophysical Einstein Minkowski modified space time metrics evaluations will evolve to understand symmetry, structure, and formation of real space geometry formed from vacuum quanta point fields' quantitative quantum general formalism theory of Iyer 
Markoulakis. Christopher O'Neill's magic square symmetry simulation results, with author's conjecture of prime numbers' factorization process operational natural mechanism aspects, combined together to author's collaborative project with Manuel Malaver's astrophysical derivational evaluation of the sense-time-space relativistic general metrics that will have means to properly account for curving or shaping of spacetime topology of a five-dimensional sensetime-space universe. These steps with steps algorithm of a quantum relativistic grand unification physics goals will be achieved by having these proceeding adequately through a series of articles highlighting them compactly.

\section{Acknowledgements}

Encouragements with group projects support of Emmanouil Markoulakis of Hellenic Mediterranean University in Greece, Manuel Malaver of Maritime University of the Caribbean in Venezuela, collaboration efforts communication with Christopher O'Neill of Cataphysics@Ireland, and perpetual project support of Engineeringinc International Operational Teknet Earth Global Platform collaborations would be appreciated highly. Author is thankful particularly to active RESEARCHGATE scientific physics forums with stimulating sessions providing collaborative ongoing debates, as well as discussions having brain storming progress.

\section{References}

[1] Rajan Iyer, and Emmanouil Markoulakis, "Theory of a superluminous vacuum quanta as the fabric of Space", accepted publication PHYSICS \& ASTRONOMY INTERNATIONAL JOURNAL, 2021. Phys Astron Int J. 2021;5(2):43-53. DOI: 10.15406/paij.2021.05.00233.

[3] Rajan Iyer, "Problem Solving Vacuum Quanta Fields", International Journal of Research and Reviews in Applied Sciences, Volume 47, Issue 1, pp. 15-25, 2021.

[2] Emmanouil Markoulakis, Antonios Konstantaras, John Chatzakis, Rajan Iyer, and Emmanuel Antonidakis, "Real time observation of a stationary magneton", Results in Physics, 2019, Vol. 15, 102793, https://doi.org/10.1016/j.rinp.2019.102793.

[4] Rajan Iyer, "Proof Formalism General Quantum Density Commutator Matrix Physics", publication at Physical Sciences \& Biophysics, Preprints 2021, 2021070267, doi:

10.20944/preprints202107.0267.v1.

[5] Rajan Iyer, "Physics Formalism Helmholtz Matrix to Coulomb Gage", process of publication in the Current Applied Physics, Preprints 2021, 2021070451, doi:

10.20944/preprints202107.0451.v1.

[6] Stefano Cremonesi, Amihay Hanany, Noppadol Mekareeyab, and Alberto Zaffaronic: Coulomb branch Hilbert series and Hall-Littlewood polynomials, Journal of High Energy Physics, Volume 2014, article id.178, 59 pp., arXiv:1403.0585, DOI:10.1007/JHEP09(2014)178. 
[7] Kirk T. McDonald, "The Helmholtz Decomposition and the Coulomb Gauge", Joseph Henry Laboratories, Princeton University, Princeton, NJ 08544, April 17, 2008; updated March 3, 2020, https://physics.princeton.edu// mcdonald/examples/helmholtz.pdf.

[8] Guillermo Arias-Tamargo, Antoine Bourget, Alessandro Pini, Diego Rodríguez-Gómez, "Discrete gauge theories of charge conjugation", Nuclear Physics B, Volume 946, September 2019, 114721, https://www.sciencedirect.com/science/article/pii/S055032131930207X https://reader.elsevier.com/reader/sd/pii/S055032131930207X?token=25C397DE4643005A0E2 77BFD0FEB90EEA3AF961776A4EEE6F086FCB908466D3D3F087E1FE949AB95827A4D67 B8C8D249.

[9] Lisa Randall, "New Mechanisms of Gauge-Mediated Supersymmetry Breaking", High Energy Physics, Nuclear Physics - Phenomenology Theory, Section B, 495, 1, 37-56, 1997, arXiv:hep-ph/9612426, https://doi.org/10.1016/S0550-3213(97)00225-3; application to special unitary group supersymmetric mass terms.

[10] Eduardo I. Guendelman, Douglas Singleton, "Scalar gauge fields", Journal of High Energy Physics, 2014 - Springer, JHEP 1405 (2014) 096, doi:10.1007/JHEP05(2014)096.

[11] C. G. Bollini, and P. Leal Ferreira, "On the Motion of a Charged Particle in the Field of a Magnetic Monopole", https://inis.iaea.org/collection/NCLCollectionStore/_Public/09/387/9387000.pdf.

[12] Carlos Bercini, Matheus Fabri, Alexandre Homrich, and Pedro Vieira, "SUSY S-matrix Bootstrap and Friends", Phys. Rev. D 101, 4, 045022, 2020, https://arxiv.org/pdf/1909.06453.pdf.

[13] "Hermitian matrix", Encyclopedia of Mathematics, EMS Press, 2001, [1994].

[14] Lucian M Ionescu, "Quantum Relativity”, General Physics, arXiv:1005.3993.

[15] John C. Hodge, "Magnetic field evolves to gravity field Part 1: Repulsion \& Part 2: Particles", 2019, http://intellectualarchive.com/?link=item\&id=2164.

[16] Katherine Jones-Smith, about identifying quasi-particles using non-Hermitian quantum mechanics, pp. 1-14, using PT quantum mechanics, Philosophical Transactions: Mathematical, Physical and Engineering Sciences, Vol. 371, No. 1989, 2013, Published by: Royal Society, https://www.jstor.org/stable/i40090204.

[17] Carl M Bender, "Making sense of non-Hermitian Hamiltonians", Reports on Progress in Physics, Volume 70, Number 6, May 2007, https:/iopscience.iop.org/article/10.1088/00344885/70/6/R03/pdf, IOP Publishing Ltd., High Energy Physics - Theory (hep-th), arXiv:hepth/0703096.

[18] Ram Mehar Singh, "Real Eigenvalue of a Non-Hermitian Hamiltonian System", Applied Mathematics, 2012, 3, 1117-1123, http://dx.doi.org/10.4236/am.2012.310164, Published Online October 2012, http://www.SciRP.org/journal/am. 
[19] B. Raes, A. W. Cummings, F. Bonell, M. V. Costache, J. F. Sierra, S. Roche, and S. O. Valenzuela, "Spin precession in anisotropic media", Physical Review B, 95, 085403, 2017.

[20] Octavian Balaci, "Connection between Gravity and Electromagnetism”, Astronomical Review, 8:4, 1-25, 2013, DOI: 10.1080/21672857.2013.11519726.

[21] Minkowski form p. 19 https://www.mv.helsinki.fi/home/syrasane/cosmo/lect2018_02.pdf.

[22] Rajan Iyer: Absolute Genesis Fire Fifth Dimension Mathematical Physics, edition 2000, Engineeringinc International Publisher with Amazon.com.

[23] Sarah Tesh, "Flash Physics: Sandra Faber wins Gruber prize, transforming magnetic monopoles, vacuum scattering revealed", COSMOLOGY NEWS, https://physicsworld.com/a/flash-physics-sandra-faber-wins-gruber-prize-transformingmagneticmonopoles-vacuum-scattering-revealed. Sarah Tesh is features editor of Physics World.

[24] Manuel Malaver, Hamed Daei Kasmaei, Rajan Iyer, Shouvik Sadhukhan , and Alokananda Kar, "A theoretical model of Dark Energy Stars in Einstein-Gauss-Bonnet Gravity", publication in progress with Applied Physics, https://arxiv.org/abs/2106.09520;

[25] Dan C. Marinescu, and Gabriela M. Marinescu, "Measurements and Quantum Information", Ch. 2, pp. 133-220, 2012; and Nilanjana Datta, "Mathematical Statistical Physics: 4.0.2 Reduced density matrix and partial trace", in Les Houches, Vol. 83. pp. 1-816, 2006, https://www.sciencedirect.com/topics/mathematics/reduced-density-operator; Mitchel Weissbluth, "Density Matrices: 13.7 Fock-Dirac Density Matrices", in Atoms and Molecules, Ch. 13, pp. 268-289, 1978; Markus Müller, ... Peter Zoller, “3.4.3 Topological Order in Density Matrices" in Advances Atomic, Molecular, and Optical Physics, 2012; Y. Fujimura, S.H. Lin, "Multiphoton Spectroscopy: II.D Density Matrix Method", in Encyclopedia of Physical Science and Technology (Third Edition), pp. 199-229, 2003; L. D. Landau, E.M. Lifshitz, "The Basic Concepts of Quantum Mechanics: §7. The density matrix”, in Quantum Mechanics: A Shorter Course of Theoretical Physics, Ch. 1, pp. 3-27, 1974; N. Chandra, and S. Parida, "Quantum Entanglement in Photon-Induced Electron Spectroscopy of Atoms and Molecules: Coulombic Density Matrix for 2-DPI", in Advances in Imaging and Electron Physics, 2016; Andreas Schadschneider, ... Katsuhiro Nishinari, "Methods for the Description of Stochastic Models: 2.6.3 Density-Matrix Renormalization Group", in Stochastic Transport in Complex Systems, Ch. 2, pp. 27-70, 2011; Nilanjana Datta, "Mathematical Statistical Physics: Quantum states and density matrices", in Les Houches, Vol. 83. pp. 1-816, 2006, https://www.sciencedirect.com/topics/mathematics/density-matrix.

[26]. Christopher C. O’Neill, "The 48 particles of the DGO Standard Model assigned vertices in the 288-cell", in Magic Squares, the Weak Force and the Higgs Mechanism, RESEARCHGATE Project: Quantum Physics, Preprint, March 2021, DOI: 10.13140/RG.2.2.23910.96321/1.. The Weak Force \& Magic Square Order 6, Preprint, May 2021, Christopher C O'Neill.

. Finite Field Construction of Odd Order Magic Squares, Preprint, Mar 2021, Christopher C O'Neill. 
.24-cell \& the Standard Model, March 2021, DOI: 10.13140/RG.2.2.16636.46725, Christopher C. O'Neill, https://www.researchgate.net/publication/349896140_24-

cell_the_Standard_Model?channel=doi\&linkId=60513680299bf173674adc0b\&showFulltext=tru e [Preprint].\}

. OCTONIONS, THE THREE FLAVOURS OF MATTER \& A NEW KIND OF SUPERSYMMETRY V. 2, DOI: 10.13140/RG.2.2.26663.98727, Christopher C. O'Neill, https://www.researchgate.net/publication/348355820_OCTONIONS_THE_THREE_FLAVOUR S_OF_MATTER_A_NEW_KIND_OF_SUPER-SYMMETRY_V_2.

. 'A Complementary Geometric Explanation of the 3 Flavours', February 2021, DOI: 10.13140/RG.2.2.12033.38240, Christopher C. O’Neill.

. 'THE GRAVITON AS 5D CHAMFERED CUBE', Christopher C. O'Neill, https://www.researchgate.net/publication/348355596_THE_GRAVITON_AS_5D_CHAMFERE D_CUBE.

. MAKING SENSE OF THE STANDARD MODEL, Christopher C. O’Neill, DOI: 10.13140/RG.2.2.34132.12163, https://www.researchgate.net/publication/349110442_MAKING_SENSE_OF_THE_STANDAR D_MODEL.

[27] Sabine Hossenfelder, "Interpretation of Quantum Field Theories with a Minimal Length Scale", Phys. Rev. D, 73, 105013, 2006, arXiv:hep-th/0603032, https://doi.org/10.1103/PhysRevD.73.105013.

[28] https://stackoverflow.com/questions/49803366/point-opacity-relative-to-depth-matplotlib3d-point-plot.

[29] Henrik J. Jensen, "Subtle relations: prime numbers, complex functions, energy levels and Riemann", https://wwwf.imperial.ac.uk/ hjjens/Riemann_talk.pdf article

[30] \{Strange Quark Star Model with Quadratic Equation of State, M Malaver, Frontiers of Mathematics and its applications 1 (1), 9-15.

. Relativistic Modeling of Quark Stars with Tolman IV Type Potential, M Malaver, International Journal of Modern Physics and Application 2 (1), 1-6.

. Charged stellar model with a prescribed form of metric function $\mathrm{y}(\mathrm{x})$ in a Tolman VII spacetime, M Malaver, World Scientific News 108, 41-52.

. Charged Anisotropic Matter with Modified Tolman IV Potential, M Malaver, Open Science Journal of Modern Physics 2 (5), 65-71.

. A THEORETICAL MODEL OF STABLE DARK ENERGY STARS, M Malaver, M Esculpi, INTERNATIONAL JOURNAL OF RESEARCH AND REVIEWS IN APPLIED SCIENCES 14 (1), 26-39. 
. Comparative Analysis of Thermal Capacity in Schwarzschild and Reissner-Nordström black holes, M Malaver, World Applied Programming 3 (2), 61-67.\}

[31] Manuel Malaver, Hamed Daei Kasmaei, Rajan Iyer, Shouvik Sadhukhan , and Alokananda Kar, "A theoretical model of Dark Energy Stars in Einstein-Gauss-Bonnet Gravity", https://arxiv.org/abs/2106.09520;

. M. Malaver, H. Kasmaei, "Mathematical Modeling of Strange Stars in 5-D Einstein-GaussBonnet Gravity", Applied Physics 4 (2), 18-35. 